\title{
Le développement durable : un défi pour l'aquaculture marine en Méditerranée
}

Helene Rey-Valette ${ }^{1}$

Jean-Paul Blancheton ${ }^{2}$

François Rene ${ }^{2}$

Jérôme Lazard ${ }^{3}$

Mathe Syndhia ${ }^{1}$

Eduardo $\mathrm{Chia}^{4}$

${ }^{1}$ Faculté de sciences économiques, CS 79606

34960 Montpellier cedex 2

$<$ fvhr@wanadoo.fr>

<syndhia.mathe@univ-montp1.fr>

${ }^{2}$ Institut français de recherche

pour l'exploitation de la mer (Ifremer),

Chemin de Maguelone,

34250 Palavas-les-Flots

$<$ Jean.paul.blancheton@ifremer.fr>

$<$ Francois.Rene@ifremer.fr>

${ }^{3}$ Centre de coopération internationale en recherche agronomique pour le développement (Cirad),

TA 30/01,

Avenue Agropolis,

34398 Montpellier cedex 5

<jerome.lazard@cirad.fr>

${ }^{4}$ Centre de coopération internationale en recherche agronomique

pour le développement (Cirad),

Département "Territoires, ressources, acteurs " (Tera)

Institut national de la recherche agronomique (Inra),

Unité mixte de recherche "Innovation »,

Bat 27 - Bureau 107,

2, place Pierre Viala,

34060 Montpellier cedex 1

<eduardo.chia@cirad.fr>

\begin{abstract}
Résumé
L'aquaculture marine méditerranéenne s'est développée à un rythme soutenu (25\% par an) et elle représente une réelle alternative à la pêche. Aujourd'hui, elle doit prendre en compte le développement durable (DD) qui constitue un véritable défi pour cette aquaculture " entrepreneuriale ". La question est donc de savoir si les entreprises aquacoles peuvent relever ce défi et avec quelles conséquences. Afin de répondre à cette question, des enquêtes ont été menées en Méditerranée. Elles visaient : i) à établir une typologie en fonction des logiques d'exploitation, des pratiques observées et des contraintes rencontrées; et ii) à étudier les représentations des acteurs de la filière vis-à-vis du DD. Elles confirment que les entreprises sont dans une logique dominée par les contraintes de marché, mais montrent aussi que leurs pratiques ne sont, pour le moment, pas trop éloignées du concept de DD. Cependant, la participation des aquaculteurs au DD passe par la mise en place d'innovations, en particulier organisationnelles, et par la constitution d'un métier qui permettrait de mettre en ouvre l'action collective nécessaire.
\end{abstract}

Mots clés : aquaculture ; développement durable ; innovation ; région méditerranéenne ; zone côtière.

Thèmes : économie et développement rural ; eau ; productions animales.

\section{Abstract \\ Sustainable development: A challenge for Mediterranean marine aquaculture}

Mediterranean aquaculture production has increased extensively over the past 10 years (25\% per year) and now constitutes a credible alternative to fishing. It now has to take the concepts of sustainability into account, placing it in a very challenging and competitive context. The current question is to know whether aquaculture companies can face up to this challenge and what the consequences will be. In order to answer this question, several surveys were carried out in the Mediterranean. They aimed at i) establishing a typology of companies based on their developmental logic, management practices and constraints; and ii) collecting information on the main stakeholders' feelings about sustainable development. These studies confirm that while such companies are complying to demanding market constraints, their current practices are not far removed from the concepts of sustainability. However, their active participation in such a dynamic of sustainable development necessitates organizational innovation and collective projects involving all the stakeholders of the sector.

Key words: aquaculture; coastal area; innovation; mediterranean region; sustainable development.

Subjects: economy and rural development; water; animal productions.

Tirés à part : H. Rey-Valette 
$\mathrm{M}$ arginale par rapport aux volumes pêchés jusque dans les années 1970, l'aquaculture a connu un développement rapide à partir du milieu des années 1980. Avec 59,4 millions de tonnes en 2004 (United Nations Food and Agriculture Organisation, 2004), elle représente presque la moitié de la production de ressources aquatiques destinées à la consommation humaine (115 Mt). Dans un premier temps, elle a été porteuse d'espoirs en matière d'avancées technologiques, de contribution nutritionnelle et de croissance, dans une logique de révolution bleue succédant à l'importante croissance de la productivité de la révolution verte. Cependant, l'élevage croissant d'espèces carnivores et l'intensification des systèmes d'élevage génèrent des impacts sur l'environnement qui limitent cette extension continue. L'aquaculture a ainsi subi plusieurs crises qui ont terni son image, telle que celle de la crevetticulture associée à la destruction d'écosystèmes sensibles (mangrove), à des itinéraires techniques mal stabilisés et à des conséquences sociales négatives sur les populations locales (Lazard et al., 2005). De même que l'agriculture a rapidement évolué vers une "révolution doublement verte" en intégrant les contraintes environnementales, le développement de l'aquaculture est à présent confronté au défi du développement durable.

Nous étudions ici les conditions de ce défi pour l'aquaculture méditerranéenne, dans le cadre d'un projet de recherche qui vise à établir une méthode générique d'analyse des facteurs de durabilité à partir d'une analyse comparative (Afrique, Asie, Europe) rendant compte de la diversité des contextes, des modes de production et des systèmes de régulation. L'analyse porte exclusivement sur l'élevage de loup et de daurade en cages en Méditerranée, activité nouvelle issue de la recherche-développement. Après un rappel historique sur la dynamique et l'origine de l'activité, on présentera en quoi et comment la question du développement durable affecte le secteur, en général et en Méditerranée. La troisième partie présente le cadre méthodologique du travail qui a été effectué en établissant la typologie des entreprises à partir de laquelle ont été programmées les enquêtes visant à analyser les pratiques et à recueillir les représentations des acteurs. Les principaux résultats de ces enquêtes font l'objet d'un quatrième point et de la discussion finale qui porte sur les conditions d'évolution vers une aquaculture durable.

\section{Bilan et dynamique de l'aquaculture méditerranéenne}

L'aquaculture marine méditerranéenne s'est développée à partir de 1972, lorsque deux scientifiques français, Gilbert Barnabé et François René ont maîtrisé la reproduction en captivité et l'élevage du loup et de la daurade (Barnabé et René, 1973). Elle était conçue à l'origine comme une réponse au plafonnement des prises halieutiques et comme une voie de reconversion pour les pêcheurs. La formation d'un secteur productif, largement subventionné, s'est faite progressivement et en marge de la pêche, ce qui a donné lieu à quelques conflits sociaux locaux (destruction des cages). La professionnalisation et le développement du secteur ont été portés par un mouvement de délocalisation progressif vers la partie orientale de la Méditerranée, qui bénéficiait de meilleures conditions physiques (température de l'eau, littoral fortement découpé) et économiques (coût de la main-d'œuvre), donnant lieu à de nombreuses opérations de joint-venture. Le poids relatif des productions françaises a rapidement décru, dès le début des années 1990, en raison notamment des difficultés d'accès aux sites, alors que les productions grecques ou turques explosaient et que les productions italiennes et espagnoles continuaient leur montée en puissance, portées par une volonté politique affichée. D'un point de vue technique, pour le grossissement c'est l'élevage en cages en mer qui devient progressivement le standard ( $87 \%$ des capacités de production), tandis que les bassins tendent à se limiter au stade de l'écloserie et de la production de juvéniles. Dès l'origine, le rôle moteur de la recherche allié à la technicité des pratiques d'élevage a conduit à la mise en place, dans une proximité tant géographique que fonctionnelle, de formations spécialisées. Bien que débouchant le plus souvent sur des emplois expatriés, celles-ci ont permis de forger une culture professionnelle commune et de construire des réseaux sociaux actifs réunissant chercheurs et anciens élèves.

Hormis l'Égypte qui se caractérise jusqu'à présent par une importante production de poissons à faible valeur (tilapia, carpe, mulet) destinée à son marché intérieur, l'aquaculture méditerranéenne est surtout une aquaculture à forte valeur (poissons carnassiers) sur le modèle de la salmoniculture. Elle se caractérise par des circuits de distribution largement dominés par la grande distribution (GMS, grandes et moyennes surfaces) et orientés vers l'exportation. Ces caractéristiques tendent à se généraliser, y compris en Égypte où on note une intensification des élevages et une diversification récente vers des espèces de poissons carnassiers destinés à l'exportation (El Sadeck, 2006). À l'origine centrée sur le loup et la daurade, l'activité s'est diversifiée à de nouvelles espèces (maigres) dont la production d'alevins a été maîtrisée à la fin des années 1990, donnant lieu à quelques milliers de tonnes de poissons marchands auxquels s'ajoutent 5000 à 6000 tonnes produites à partir d'alevins de pêche en Égypte. Par ailleurs, de nouvelles pratiques apparaissent avec le grossissement de thon qui, 20 ans après, répond aux préoccupations initiales de complémentarité avec la pêche. Cette production de thon d'aquaculture, démarrée à la fin des années 1990, se stabilise actuellement autour de 20000 à 30000 tonnes en fonction des captures halieutiques qui sont à la fois aléatoires et plafonnées par des quotas annuels visant à réguler la pêche. En Méditerranée, l'aquaculture est principalement confrontée à des contraintes de marché et de compétitivité du fait de ses choix initiaux en faveur d'espèces à forte valeur ainsi qu'à des problèmes de disponibilité en sites, en raison de l'importante concurrence spatiale avec les autres activités, notamment le tourisme. On note une baisse des prix de $35 \%$ en 2002 qui résulte d'un effet de "ciseau " à la suite, d'une part de l'obligation commerciale de différenciation de l'origine (pêche ou élevage) des produits (University of Stirling, 2004) et, d'autre part, de la forte progression des productions grecques et turques à faibles coûts. Dans le même temps, la forte pression foncière engendrée par la vocation résidentielle des côtes méditerranéennes (Benoît et Comeau, 2005) va conduire à un durcissement de la réglementation. La mise en place de conditions restrictives pour l'accès à l'espace conduit à un tarissement des nouvelles installations, notamment dans les pays initiateurs. Ainsi, l'aquaculture en France est soumise à une obligation d'enquêtes publiques liées aux études d'impacts environnementaux dans le cadre des procédures "Installation classée pour l'environnement (ICPE)». Face au risque de la multiplication des procès (juridiciarisation de la société), le représentant des 
pouvoirs publics (le préfet), seul responsable in fine des autorisations, tend à appliquer le principe de précaution et à refuser toute nouvelle installation. Parallèlement, la création de droits à produire par l'octroi de licences définissant des quotas annuels de production conformes aux capacités d'accueil des milieux et l'exigence nouvelle d'une acceptation des nouveaux sites de production par la société civile riveraine via des enquêtes publiques, restreignent le développement aux sites existants. Il en résulte des dépassements de quotas de production et, par là, des capacités de charge des milieux du fait des contraintes de productivité des entreprises. La conjugaison de ces facteurs a donc conduit à une concentration du secteur par rachat d'entreprises existantes et par délocalisation vers des pays où ces contraintes d'accès n'existent pas encore et qui pour la plupart bénéficient également de plus faibles coûts de main-d'œuvre.

\section{L'aquaculture méditerranéenne face aux initiatives en faveur d'une aquaculture durable}

Dans le contexte actuel de développement d'initiatives en faveur du développement durable, on peut s'interroger sur la possibilité et la capacité du secteur, compte tenu de la structure capitalistique forgée par une dynamique très concurrentielle entre les unités, à s'adapter à ce nouveau référentiel. Bien que l'aquaculture soit mentionnée dans le Code de conduite pour les pêches responsables établi par l'Organisation des Nations unies pour l'agriculture et l'alimentation (FAO) en 1995, l'application spécifique des objectifs de développement durable à l'aquaculture ne sera formalisée qu'en 1997 par la directive sectorielle de la FAO. L'analyse des initiatives menées dans ce domaine en fonction de leur origine institutionnelle et de leur degré de contrainte (tableau 1) montre la prépondérance des démarches normatives portées par des actions émanant de la recherche, des institutions internationales (Union inter-

Tableau 1. Typologie des modes d'action en faveur de la durabilité.

Table 1. Typology of the means for fostering sustainability.

\begin{tabular}{|c|c|c|}
\hline & Initiative décentralisée & $\begin{array}{l}\text { Initiative centralisée } \\
\text { (États ou organisations } \\
\text { internationales) }\end{array}$ \\
\hline Faible contrainte & $\begin{array}{l}\text { Recommandations } \\
\text { Déclaration }\end{array}$ & $\begin{array}{c}\text { Convention } \\
\text { Plans d'actions }\end{array}$ \\
\hline Forte contrainte & $\begin{array}{c}\text { Codes de conduites } \\
\text { Guides de pratiques } \\
\text { Chartes } \\
\text { Labels et certifications }\end{array}$ & $\begin{array}{c}\text { Programmes d'actions } \\
\text { Stratégies territoriales } \\
\text { Lois }\end{array}$ \\
\hline
\end{tabular}

Source : Mathé et al., 2006.

nationale de conservation de la nature (UICN), FAO, Union européenne) ou des syndicats professionnels (Clément, 2006 ; Mathé et al., 2006).

Si dans d'autres secteurs les initiatives issues des entreprises dans le cadre de la responsabilité sociale des entreprises (RSE) sont fréquentes, cette voie est marginale en aquaculture. L'existence de plans globaux HACCP (hazard analysis critical control point) se limite à quelques pays tels les États-Unis, l'Australie, le Chili, la Norvège, la Nouvelle-Zélande et la Thailande, et ne concerne que certaines espèces. Ce sont donc essentiellement des initiatives de construction de référentiels généraux internationaux qui caractérisent l'application du développement durable à l'aquaculture. L'appropriation de ces référentiels à des échelles locales qui constituent en général une seconde phase, reste largement à construire. Plusieurs actions, ciblant notamment la crevetticulture, méritent cependant d'être mentionnées pour leurs effets structurants, même si elles n'ont pas eu d'applications concrètes en Méditerranée. Il s'agit en premier lieu du Programme Aquaculture responsable de la Global Alliance Aquaculture (GAA) lancé dès 1996 et visant à établir un code de pratique en faveur d'une aquaculture responsable. Divers types d'actions ont ensuite été mis en œuvre à différentes échelles spatiales et émanant de multiples origines institutionnelles (syndicats, organisations internationales, organisations non gouvernementales (ONG) ou institutions de recherche), dont récemment l'action européenne Consensus qui a permis d'élaborer 78 indicateurs rendant compte des différentes facettes de la durabilité de l'activité. En Méditerranée, la prise en compte du développement durable s'effectue dès 1994 dans le cadre du Programme d'action méditerranéen (PAM, 2006) et se concrétise par la mise en place de la Commission méditerranéenne du développement durable en 1996, par la construction d'indicateurs de développement durable pour les régions côtières de Méditerranée en 1999, et enfin par l'élaboration d'un bilan et d'une stratégie d'action (Benoît et Comeau, 2005). Concernant l'aquaculture, peu d'actions sont recensées à l'échelle de la Méditerranée (Mathé et al., 2006), en dehors de groupes de travail organisés à l'instigation de l'UICN et de la Fédération européenne des producteurs aquacoles (FEAP) à Alger en 2005 et à Las Palmas (Canaries) en 2006 (Union internationale de conservation de la nature, 2005a ; Union internationale de conservation de la nature, 2005b). Les producteurs européens sont cependant concernés par les actions menées à des échelles nationales ou européennes, comme par exemple le plan d'action du Centre interprofessionnel des produits de l'aquaculture (Cipa) pour la France ou le code de conduite de la FEAP, voire entre producteurs locaux comme par exemple les producteurs de Corse ou à l'occasion d'actions de gestion intégrée du littoral (étude d'implantation d'un lotissement aquacole en mer par la communauté d'agglomérations ToulonProvence-Méditerranée).

\section{Structure fonctionnelle du secteur et cadre méthodologique des enquêtes réalisées}

À partir de la bibliographie disponible, notamment l'étude récente de l'Université de Stirling et les rapports nationaux "National Aquaculture Sector Overview" 


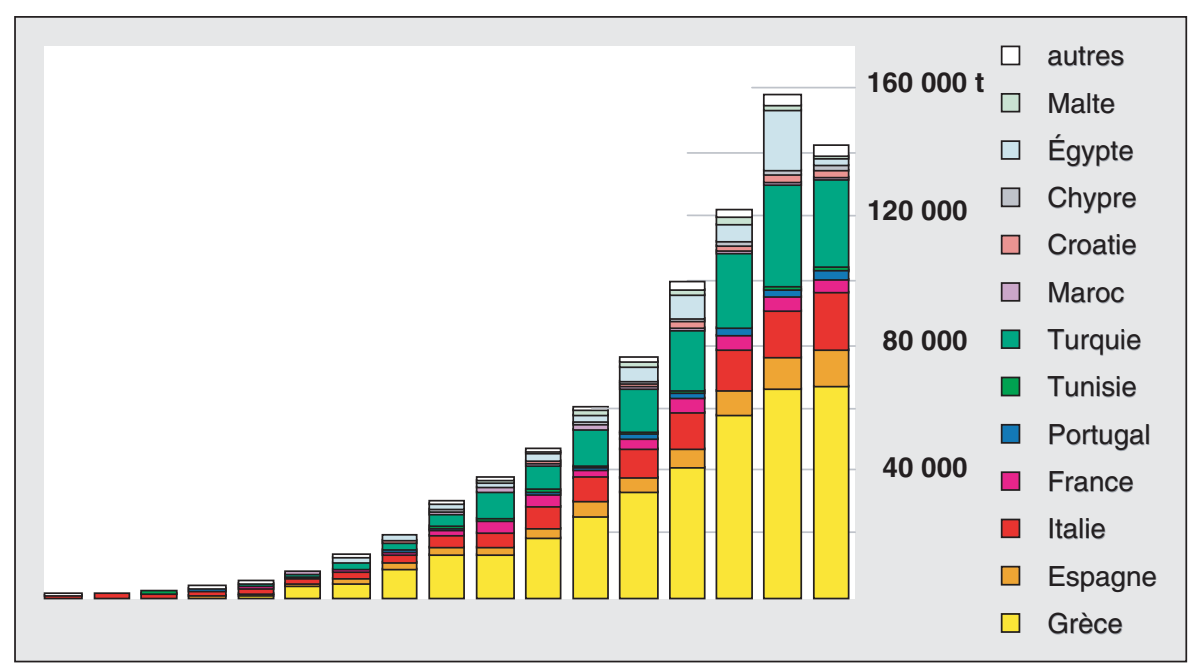

Figure 1. Évolution des productions de loup et de daurade de 1986 à 2002.

Figure 1. Evolution of sea bass and sea bream production from 1986 to 2002.

Source : données FAO, 2004.

(NASO) des correspondants du Système d'information pour la promotion de l'aquaculture en Méditerranée (SIPAM), on a estimé que la production du secteur s'élevait en 2005 à environ 250000 tonnes pour un volume déclaré (du fait des sous-déclarations fiscales et des plafonds de quotas résultats des droits à produire) de 180000 tonnes (source Aquamedia ${ }^{1}$ ). La distribution spatiale de la production finale (animaux marchands), essentiellement réalisée en cages (87\%), témoigne d'une forte concentration au profit de quatre pays : la Grèce, la Turquie, l'Italie et l'Espagne (figure 1). La production d'alevins est aussi très concentrée, la Grèce, avec 270 millions d'alevins en 2003 arrivant largement en tête devant l'Italie avec 90 millions d'alevins, la Turquie, l'Espagne et la France. Au total, le chiffre d'affaires de l'élevage (écloserie et grossissement) pour le loup et la daurade peut être estimé à environ 900000 euros (Mathé et al., 2006).

Afin d'illustrer la concentration du secteur, les figures suivantes caractérisent les profils des principaux pays producteurs en fonction des différentiels de contribution des classes de taille d'entreprises aux productions nationales (figure 2) et de la répartition des effectifs d'entreprises par classes de taille (figure 3).

À l'exception de la Turquie, ces données confirment la concentration de l'aquacul-

\footnotetext{
${ }^{1}$ Réseau $d$ 'information de la Fédération européenne des producteurs aquacoles (FEAP). www.feap.info/feap/
} d'experts. Quatre grands types d'entreprises ont été définis en fonction de leur taille
(> 500 tonnes et $<100$ tonnes), de leur structure de capital et des circuits de commercialisation (tableau 2). Outre ces types, on trouve des entreprises de taille moyenne (entre 100 et 500 tonnes), mais qui constituent souvent une forme intermédiaire dans le cycle de vie de l'entreprise et qu'il n'était donc pas pertinent de retenir en tant qu'archétype.

Cette typologie a permis d'échantillonner les unités à enquêter et de structurer l'analyse des pratiques au sein des entreprises. Les données recueillies résultent de deux types d'enquêtes :

- des enquêtes semi-directives auprès des entreprises de production (enquête 1) qui permettent d'analyser leur fonctionnement, leur stratégie et d'identifier les systèmes de pratiques ;

- des enquêtes sur les représentations (enquête 2) de la filière, son devenir et la manière dont l'aquaculture contribue ou non au développement durable, réalisées auprès de l'ensemble des acteurs parties prenantes concernés par l'aquaculture. Il s'agit d'identifier les principes structurants auxquels les acteurs sont sensibles et les facteurs de durabilité de l'activité.

Les difficultés d'accès aux entreprises aquacoles méditerranéennes ont conduit à recentrer les enquêtes sur deux pays représentatifs de deux grands contextes de production, à savoir la France comme archétype des pays initiateurs en Méditerranée occidentale et Chypre en tant qu'exemple des nouveaux pays producteurs de Méditerranée orientale. Au total, 61 enquêtes ont été réalisées auprès des

producteurs et des acteurs parties prenantes

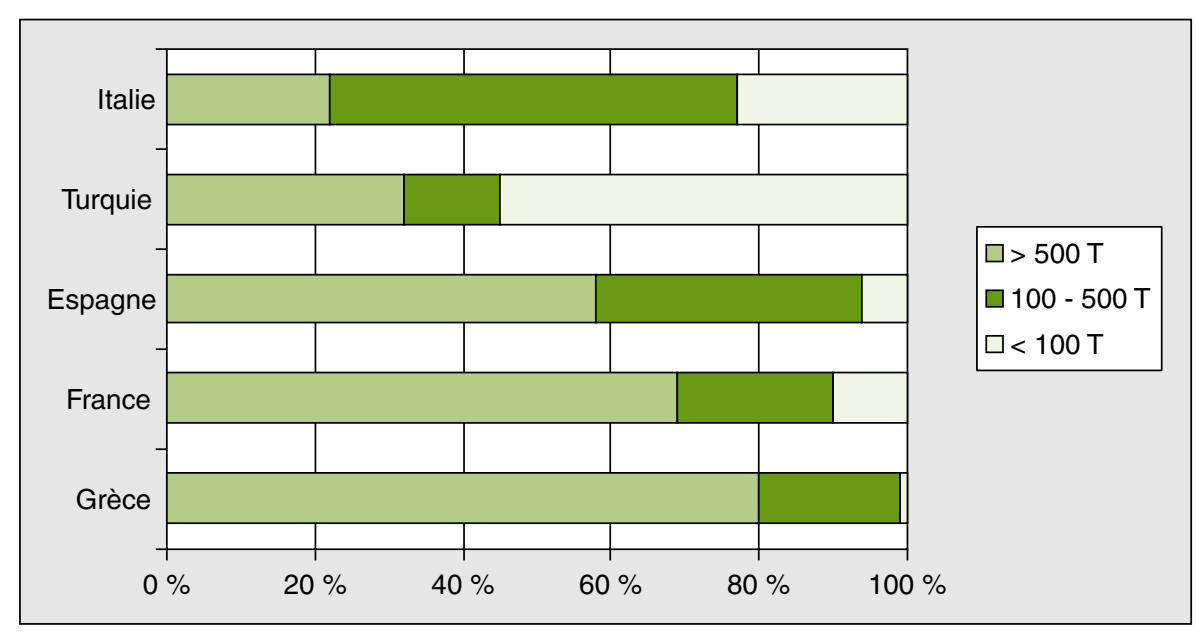

Figure 2. Répartition des productions nationales en fonction des tailles d'entreprises (2004).

Figure 2. Distribution of national production according to company size (2004). Source : d'après données University of Stirling, 2004. 


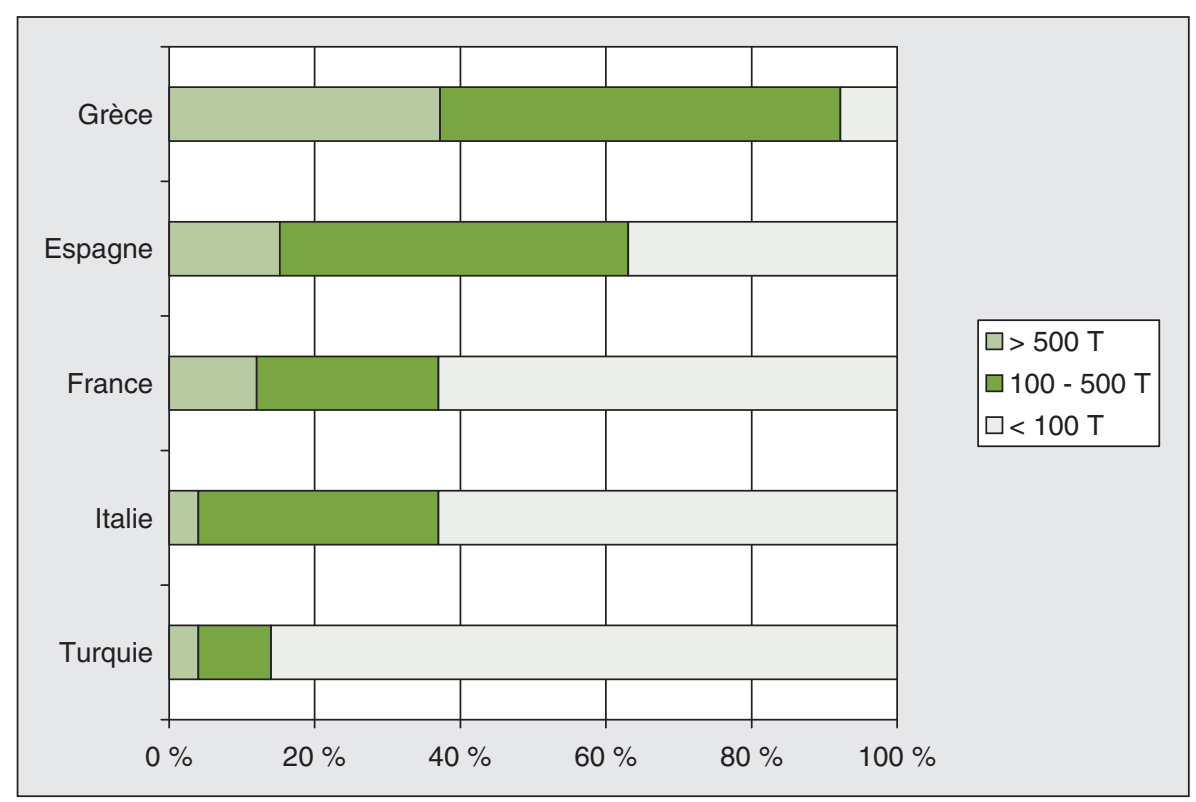

Figure 3. Répartition des entreprises en fonction des tailles (2004).

Figure 3. Distribution of companies according to size (2004).

Source : d'après données University of Stirling, 2004.

(acteurs institutionnels et intervenant de la filière (tableaux 3 et 4, figure 4)). La prise en compte des représentations des acteurs parties prenantes constitue une condition à la mise en œuvre du développement durable, qui introduit des interactions entre les stratégies individuelles et collectives, et oblige ainsi à renouveler les approches des exploitations agricoles dont les frontières deviennent plus difficiles à cerner (Gafsi, 2006). Le dispositif d'enquêtes mis au point permet une bonne représentation des secteurs productifs (plus de $80 \%$ des unités) et des acteurs liés à l'activité dans les deux pays étudiés.

\section{Représentations des acteurs et voies d'évolution vers I'aquaculture durable}

Les premiers résultats confirment le caractère dominant de la logique de marché, mais révèlent le fait que les pratiques des entreprises ne sont pas trop éloignées des conditions de développement durable.
Cependant, le type de réponse à la baisse des prix par une course aux gains de productivité au travers de stratégies d'agrandissement et d'intensification pourrait tendre à un éloignement des conditions de l'aquaculture durable. L'analyse des représentations des acteurs a été réalisée à partir de questions ouvertes où aucune suggestion de réponse n'était proposée. Le traitement des arguments évoqués et des terminologies utilisées a été ensuite réalisé à partir d'un regroupement progressif et raisonné des réponses relevant de notions similaires.

On note la prééminence de la logique économique pour l'ensemble des producteurs, quelle que soit la taille de l'entreprise. Ceux-ci caractérisent en effet l'activité par sa capacité à "fournir des poissons de haute qualité à des prix raisonnables " c'est-à-dire en termes d'offre et de marché. D'autres fonctions telles que la participation au développement économique local ou le fait de suppléer la pêche ont aussi été évoquées tant par les acteurs institutionnels parties prenantes que par les producteurs. Tandis que certains acteurs dénoncent les possibilités d'impacts environnementaux négatifs en termes de pollution ou de consommation de ressources (farines et espaces), les producteurs insistent sur les effets positifs de l'activité sur le milieu en citant notamment leur rôle de sentinelle de la qualité des eaux et l'effet récif généré par les élevages en mer.

Concernant les impacts possibles du développement durable, les attentes et perspectives évoquées par les acteurs font ressortir une représentation plutôt positive de ces impacts, comme en témoigne la figure 5 qui illustre les représentations des acteurs, tous types confondus à propos du développement durable. En effet, si pour un tiers des acteurs il n'y pas

\section{Tableau 2. Typologie et caractéristiques des entreprises d'aquaculture méditerranéenne.}

Table 2. Typology and characteristics of Mediterranean aquaculture units.

\begin{tabular}{|c|c|c|c|c|c|c|c|c|c|c|c|c|c|c|c|}
\hline Type d'entreprises & $\begin{array}{c}\text { Capital } \\
\text { familial }\end{array}$ & $\begin{array}{r}\text { Capital } \\
\text { externe }\end{array}$ & $\begin{array}{r}\text { Multi- } \\
\text { sites }\end{array}$ & $\begin{array}{c}\text { Multi- } \\
\text { activités }\end{array}$ & $\begin{array}{c}\text { Plusieurs } \\
\text { stades }\end{array}$ & $\begin{array}{r}\text { Plusieurs } \\
\text { produits }\end{array}$ & $\begin{array}{l}\text { Plusieurs } \\
\text { espèces }\end{array}$ & $\begin{array}{c}\text { Savoir-faire } \\
\text { familial }\end{array}$ & $\begin{array}{r}\text { Diplôme } \\
\text { spécifique }\end{array}$ & $\begin{array}{c}\text { Savoir-faire } \\
\text { pratique }\end{array}$ & $\begin{array}{c}\text { Marché } \\
\text { local }\end{array}$ & $\begin{array}{l}\text { Marché } \\
\text { national }\end{array}$ & $\begin{array}{c}\text { Marché à } \\
\text { l'export }\end{array}$ & $\begin{array}{l}\text { Présence } \\
\text { de labels }\end{array}$ & $\begin{array}{c}\text { Forte } \\
\text { régulation }\end{array}$ \\
\hline $\begin{array}{l}\text { G1: } \\
\text { taille importante et } \\
\text { capitaux familiaux }\end{array}$ & $x$ & & $x$ & & $x$ & $x$ & $x$ & $x$ & $x$ & $x$ & & $x$ & $x$ & $x$ & $x$ \\
\hline $\begin{array}{l}\text { G2: } \\
\text { taille importante et } \\
\text { capitaux externes }\end{array}$ & & $x$ & $x$ & & $x$ & $x$ & $x$ & & $x$ & $x$ & & & $x$ & $x$ & $x$ \\
\hline $\begin{array}{l}\text { P1 : } \\
\text { petite taille } \\
\text { et forte valorisation }\end{array}$ & $x$ & $\times$ & & & & & $\times$ & $x$ & $x$ & $x$ & $\times$ & $\times$ & & $x$ & $x$ \\
\hline $\begin{array}{l}\text { P2: } \\
\text { petite taille } \\
\text { et faibles coûts }\end{array}$ & $x$ & & & $x$ & & & $x$ & $x$ & & $x$ & & & $\times$ & & \\
\hline
\end{tabular}


Tableau 3. Enquêtes réalisées en Méditerranée, par type d'enquête.

Table 3. Surveys carried out in the Mediterranean region, by type of surveys.

\begin{tabular}{lc}
\hline & Total \\
\hline Enquête 1 : systèmes productifs & $\mathbf{2 1}$ \\
Enquête 2a : perception des acteurs non institutionnels & $\mathbf{9}$ \\
de la filière & $\mathbf{2 4}$ \\
Enquête 2b : perception des acteurs institutionnels & $\mathbf{7}$ \\
Enquête 2c : perception des producteurs & $\mathbf{6 1}$ \\
Total
\end{tabular}

\section{Tableau 4. Enquêtes réalisées en Méditerranée, par type d'acteur.}

Table 4. Surveys carried out in the Mediterranean region, by type of stakeholders.

\begin{tabular}{lcr}
\hline \multicolumn{1}{c}{ Groupes d'acteurs } & Effectifs & \% \\
\hline Amont & 3 & 7,5 \\
Aval & 4 & 10,0 \\
Administration & 2 & 5,0 \\
Services administratifs & 9 & 22,5 \\
Recherche & 5 & 12,5 \\
Autres institutions & 8 & 20,0 \\
Secteur (dont 2 services de l'entreprise) & 9 & $\mathbf{2 2 , 5}$ \\
Total & $\mathbf{4 0}$ & $\mathbf{1 0 0 , 0}$
\end{tabular}

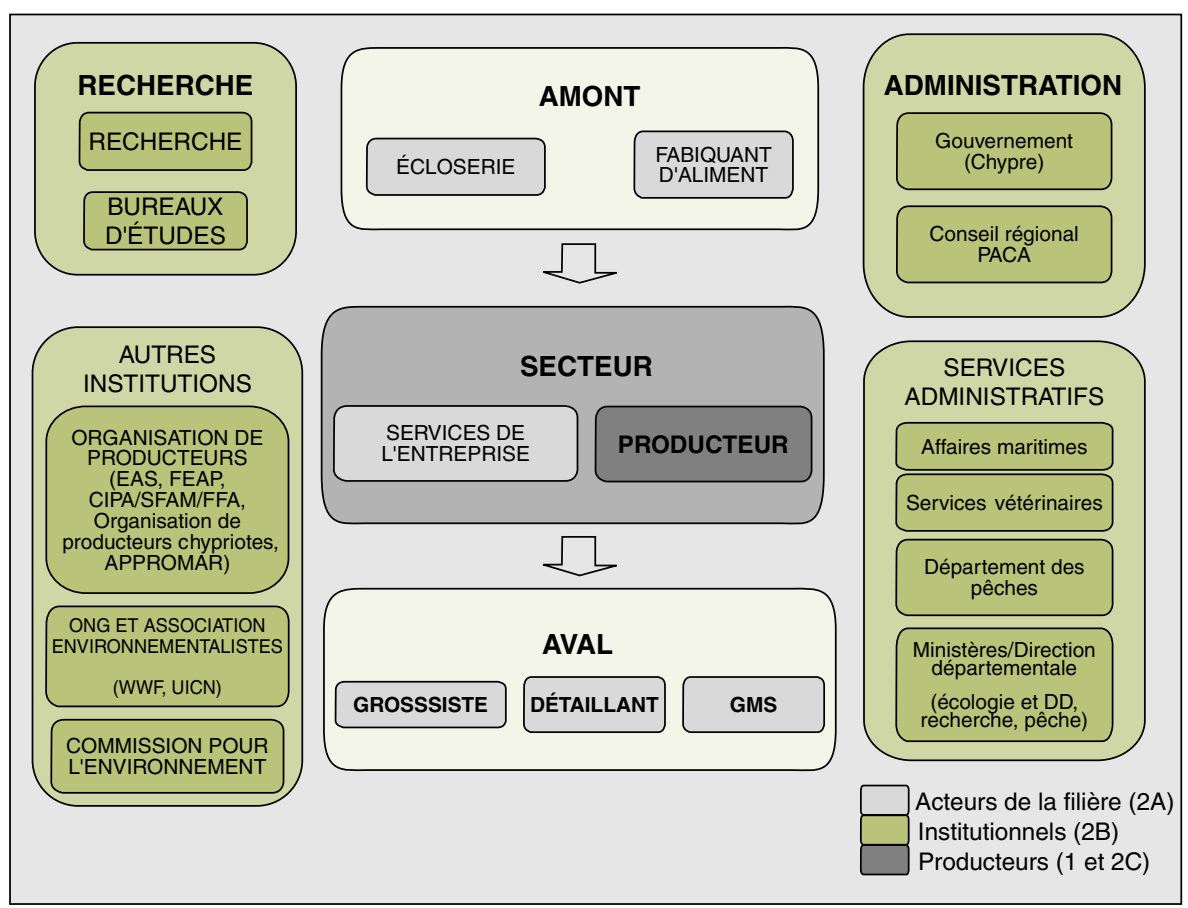

Figure 4. Présentation des types d'acteurs enquêtés (en France et à Chypre).

Figure 4. Categories of surveyed stakeholders (in France and in Cyprus). de changements significatifs, les arguments évoqués par les autres relèvent majoritairement d'effets positifs : les seuls aspects contraignants sont un renforcement des difficultés économiques (6\%) ou des risques de durcissement des réglementations (2\%).

Au-delà de ces arguments généraux, les types de représentations ont été étudiés en détail en fonction des domaines dans lesquels se décline le développement durable. Dans le domaine environnemental, l'ensemble des réponses converge et souligne la nécessité d'améliorer la compatibilité de l'activité à son milieu en s'adaptant à la capacité de charge de celui-ci. Pour la plupart, cette condition suppose la maîtrise des effluents et des rejets, quelques-uns évoquant aussi l'intégration dans le paysage. Dans le domaine social, ce sont surtout les possibilités d'amélioration de l'image de l'activité et par là de son insertion locale qui sont mentionnées (figure 6), tandis que pour les aspects économiques un plus grand nombre d'impacts possibles a été évoqué, dont principalement l'amélioration de la qualité des produits (figure 7).

Les réponses obtenues auprès des acteurs institutionnels à propos des modes de mise en œuvre du développement durable témoignent d'une certaine connaissance des implications concrètes de celui-ci mais d'une faible conscience du fait qu'il puisse être un outil pour orienter la filière (figure 8). On observe une diversité de moyens, dont la nécessité de maintenir une diversité des types d'exploitations. La nature des actions évoquées témoigne de l'importance des mesures de communication, formations et encadrement qui relèvent d'une meilleure gouvernance du secteur. Or les enquêtes témoignent de la faible structuration professionnelle locale du secteur et de l'absence d'organisation interprofessionnelle en dehors des échelles nationales, situation qui constitue une contrainte pour la mise en place de démarches collectives en faveur de la durabilité et plus généralement pour la participation et la représentation du secteur à des politiques locales de gestion intégrée des zones côtières (GIZC) visant le développement durable des zones littorales.

Enfin, concernant les perspectives d'évolution du secteur, les représentations sont plus partagées. La figure 9 témoigne en effet du fait que, pour près de la moitié des acteurs, c'est le modèle actuel d'exploitation de grande taille qui reste dominant, ce qui relativise la perception 


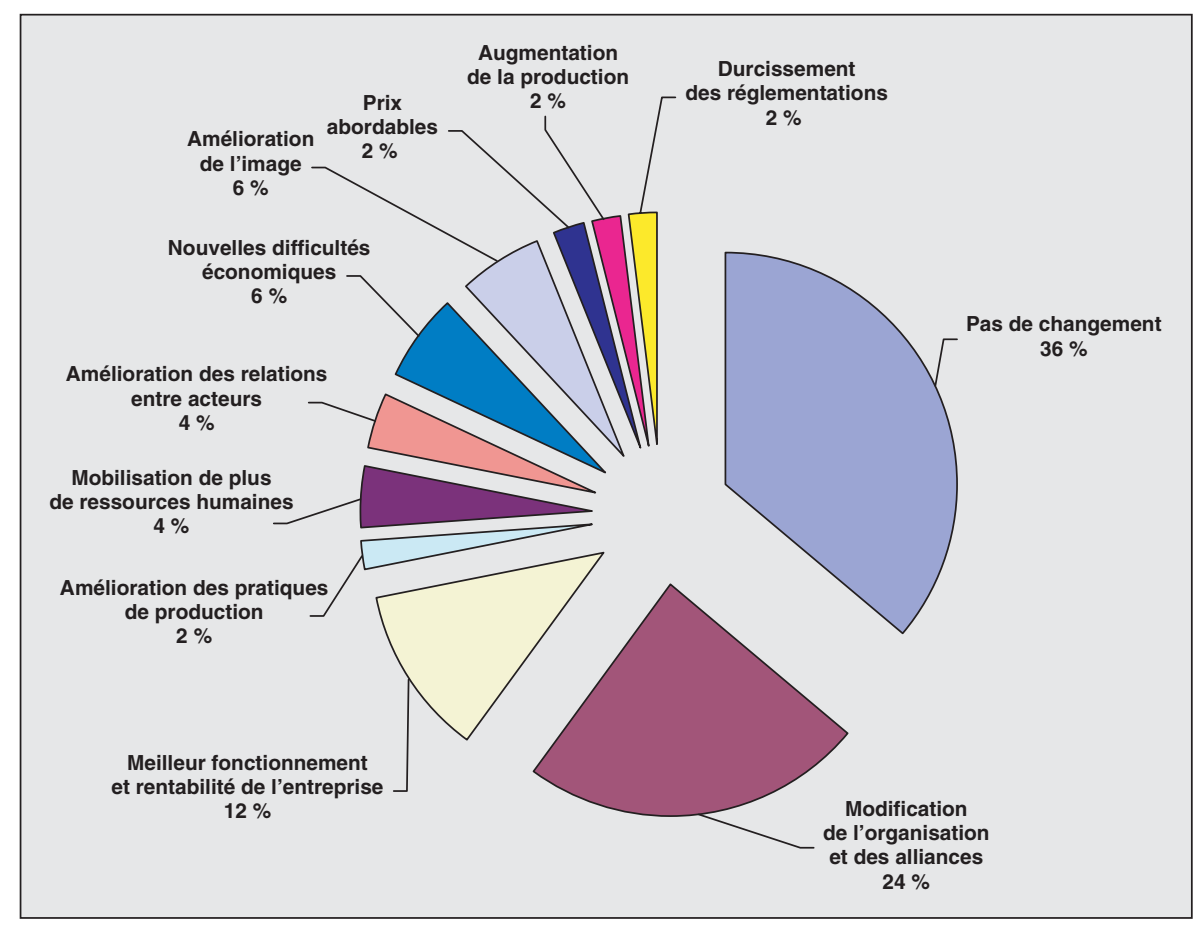

Figure 5. Représentation des effets du développement durable pour l'aquaculture.

Figure 5. Representation of the consequences of sustainable development for aquaculture. Source : enquêtes EVAD, France et Chypre été 2006.

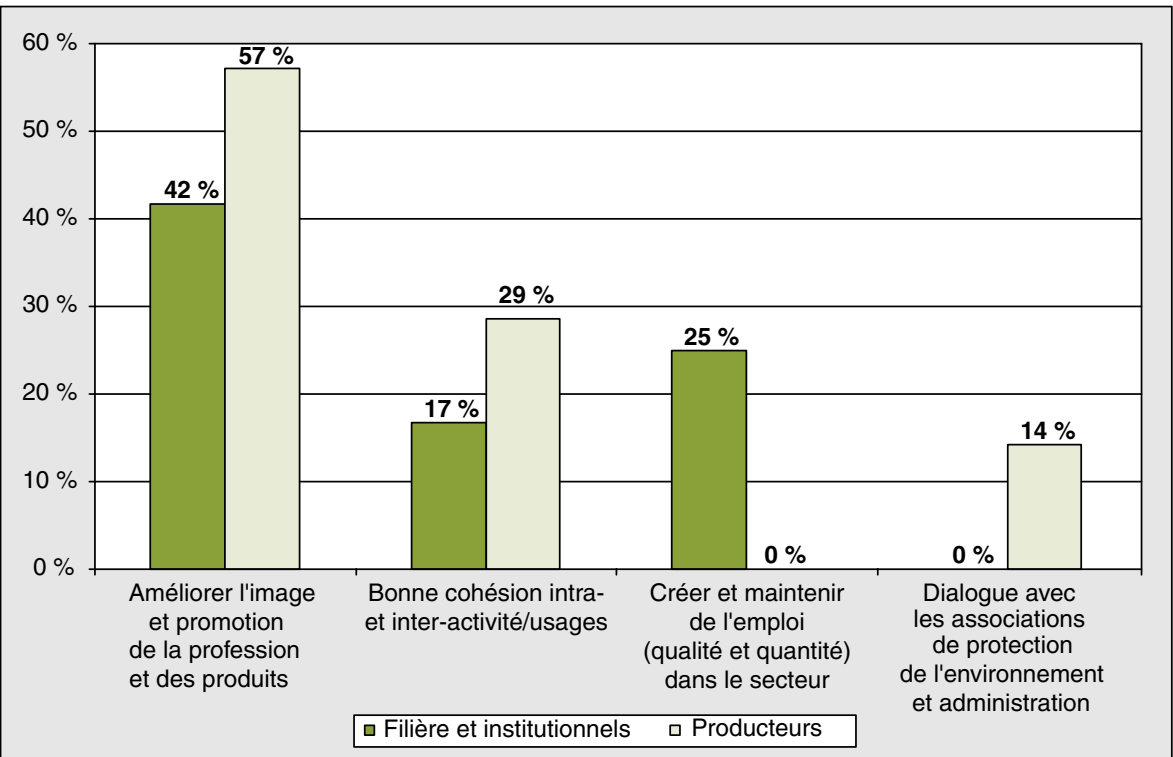

Figure 6. Représentations des conséquences sociales du développement durable.

Figure 6. Representation of the social consequences of sustainable development. Source : enquêtes EVAD, France et Chypre été 2006. positive et l'intérêt affiché pour le développement durable. En revanche, les évolutions techniques paraissent plus ouvertes avec la domination du modèle actuel des cages en mer qui pourrait être révisée à l'avenir au profit, notamment, d'une aquaculture off shore (figure 10).

\section{Quels axes}

\section{et quelles modalités pour une aquaculture littorale durable?}

À partir des résultats des enquêtes et compte tenu des évolutions institutionnelles en ouvre au niveau de la gestion intégrée du littoral, il convient de s'interroger sur les stratégies d'adaptation (innovations techniques et/ou organisationnelles) et leurs limites. Face à l'accroissement des contraintes deux grandes orientations se dessinent. L'évolution vers une aquaculture au large constitue une réponse qui s'inscrit dans le prolongement des logiques précédentes qui visent à un contournement des contraintes en se déplaçant vers des espaces moins régulés. En revanche, une aquaculture durable et intégrée suppose une transformation des dynamiques de façon à maintenir une diversité des types et des tailles d'entreprises et à favoriser leur intégration dans les politiques territoriales de gestion intégrée. Comme l'illustre l'évolution halieutique passée où la réponse aux conflits et à la surexploitation des stocks a longtemps consisté en un élargissement des zones de pêche, la délocalisation vers de nouveaux espaces de production off shore ne peut constituer à elle seule une réponse satisfaisante. Il est nécessaire de penser l'activité autrement, non seulement au niveau individuel des pratiques d'exploitation mais collectivement en cherchant les conditions de l'intégration territoriale de l'activité, notamment au travers des politiques de GIZC. Celles-ci recherchent les conditions d'articulation des politiques sectorielles dans des projets concertés de développement durable des territoires littoraux. Cette entrée par l'espace permet d'intégrer les piliers du développement durable et de faciliter l'appropriation par les acteurs des objectifs de celui-ci en fonction des enjeux locaux. On ne 


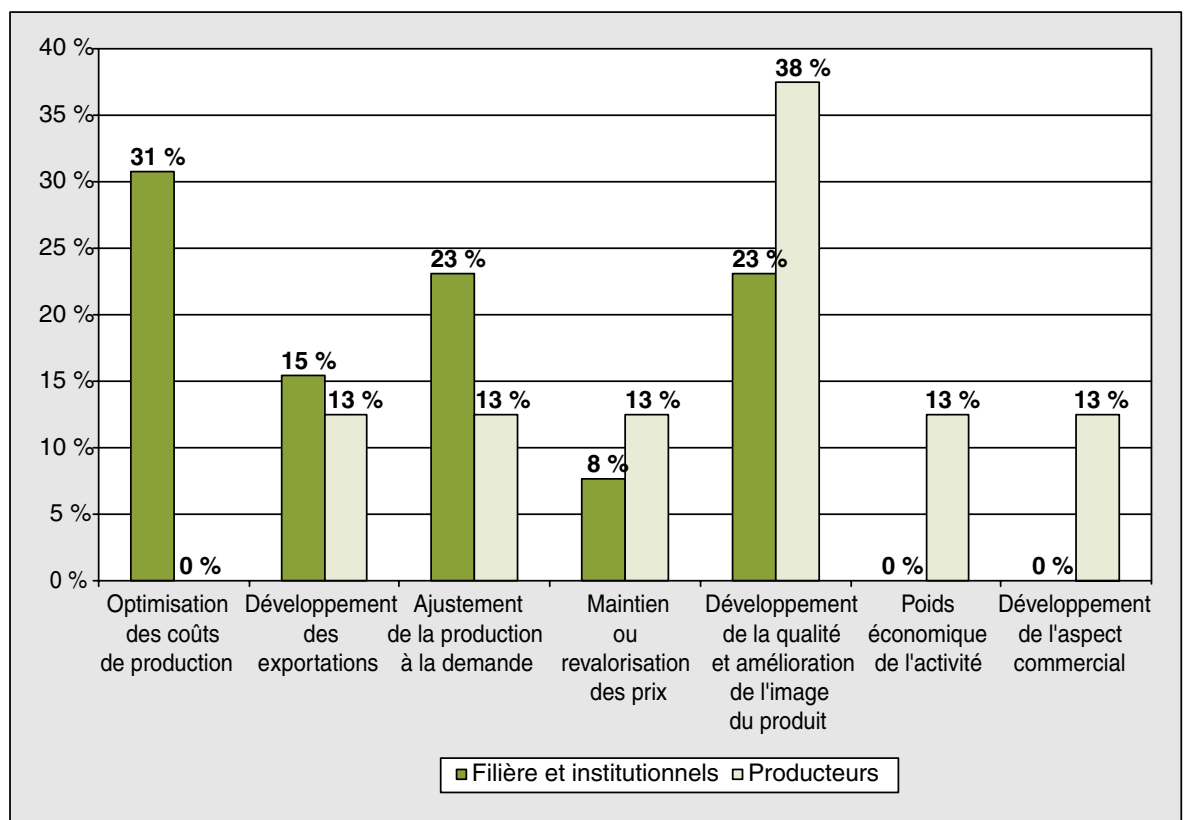

Figure 7. Représentations des conséquences économiques du développement durable.

Figure 7. Representation of the economic consequences of sustainable development. Source : enquêtes EVAD, France et Chypre été 2006.

reviendra pas sur les définitions et conditions d'application territoriale et institutionnelle de la GIZC, étudiées par ailleurs (Rey-Valette et Roussel, 2006) et déjà expérimentées en Méditerranée comme en témoigne le bilan effectué par le Plan d'action pour la Méditerranée (Benoît et Comeau, 2005). Il convient d'insister ici sur les conditions de cette articulation entre aquaculture durable et politiques de GIZC. Il ne s'agit plus seulement de promouvoir des entreprises aquacoles durables mais aussi de veiller à la durabilité des territoires où l'aquaculture est implantée. Cette condition est d'autant plus importante que les zones côtières constituent des zones sensibles, telles les zones humides ou lagunaires qui relèvent souvent du réseau Natura $2000^{2}$. Cette durabilité des territoires ressort de politiques publiques d'aménagement qui sont mises en ouvre par des gestionnaires locaux, notamment les collectivités territoriales. Il revient alors aux acteurs "aquacoles", pour mieux s'insérer dans ces dispositifs de gestion, d'intégrer ces nouvelles logiques, notamment en diversifiant les indicateurs de développement durable relatifs à leur activité et en s'insérant au mieux dans les dispositifs institutionnels d'aménagement. Il faudra pour appréhender les sources des dysfonctionnements à l'échelle de l'écosystème en tenant compte des multiples interactions entre composantes, dépasser le stade de la cohabitation des usages, des mesures d'aménagement par spécialisation spatiale des activités (pour réduire les conflits) et des modes de gestion axés sur la régulation des effets sur l'environnement. Les politiques de GIZC relèvent d'une logique de contrat et de projet de territoire associant plusieurs partenaires et impliquant le recours à des processus de concertation autour d'objectifs communs. Alors que l'Union européenne prépare une directive cadre pour l'application de la GIZC et que de nombreuses initiatives nationales sont en cours, tel le programme expérimental mis en ouvre en France (Diact, 2006), il est important que la filière aquacole s'organise et s'inscrive dans une stratégie pro active vis-àvis de ces régulations à venir. Pour cela, il est important de développer tout à la fois une conscience sectorielle et une structuration à l'échelle du Bassin méditerranéen.

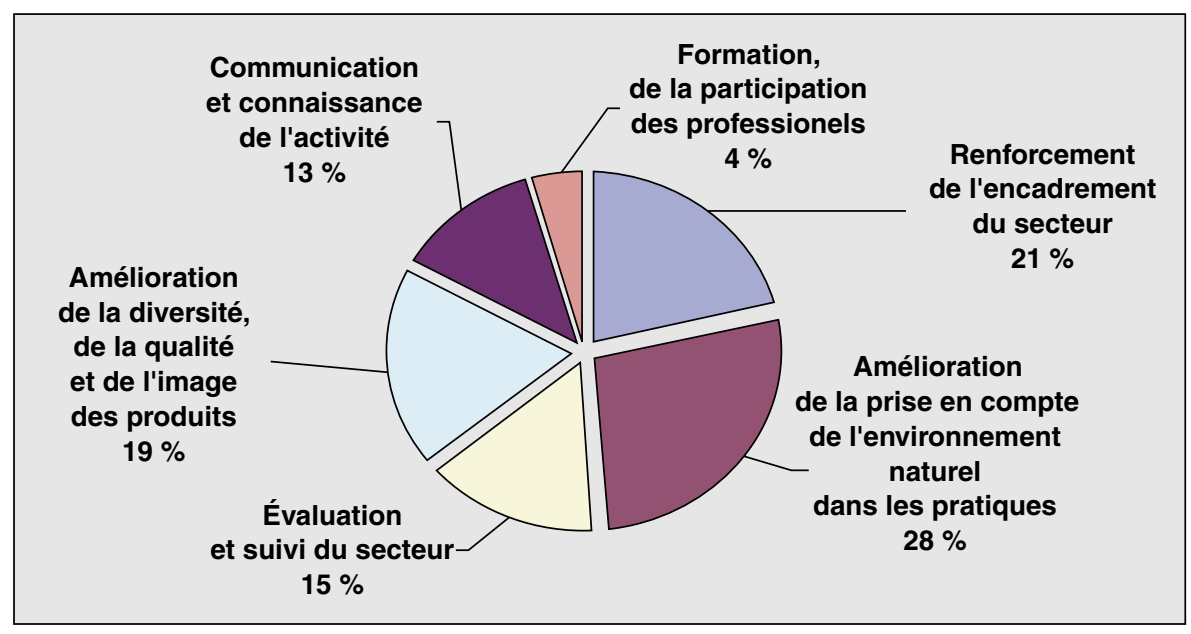

Figure 8. Modes de mise en œuvre du développement durable dans l'aquaculture.

Figure 8. Ways of implementing aquaculture sustainable development. Source : enquêtes EVAD, France et Chypre été 2006.

\section{Conclusion}

L'aquaculture méditerranéenne est confrontée à de multiples contraintes, notamment en termes d'intégration dans les territoires littoraux soumis à des pressions d'appropriation de plus en plus conflictuelles et intenses. Or, l'utilisation de cet espace ne peut plus s'imaginer que coconstruite et cogérée dans un souci de maximisation des synergies entre activités. Il s'agit donc de réviser les logiques de course aux gains de productivité résultant de la concurrence commerciale très vive, pour aller vers des stratégies plus axées sur la qualité en favorisant une

\footnotetext{
${ }^{2}$ Réseau Natura: réseau écologique euro-
} péen de sites naturels. 


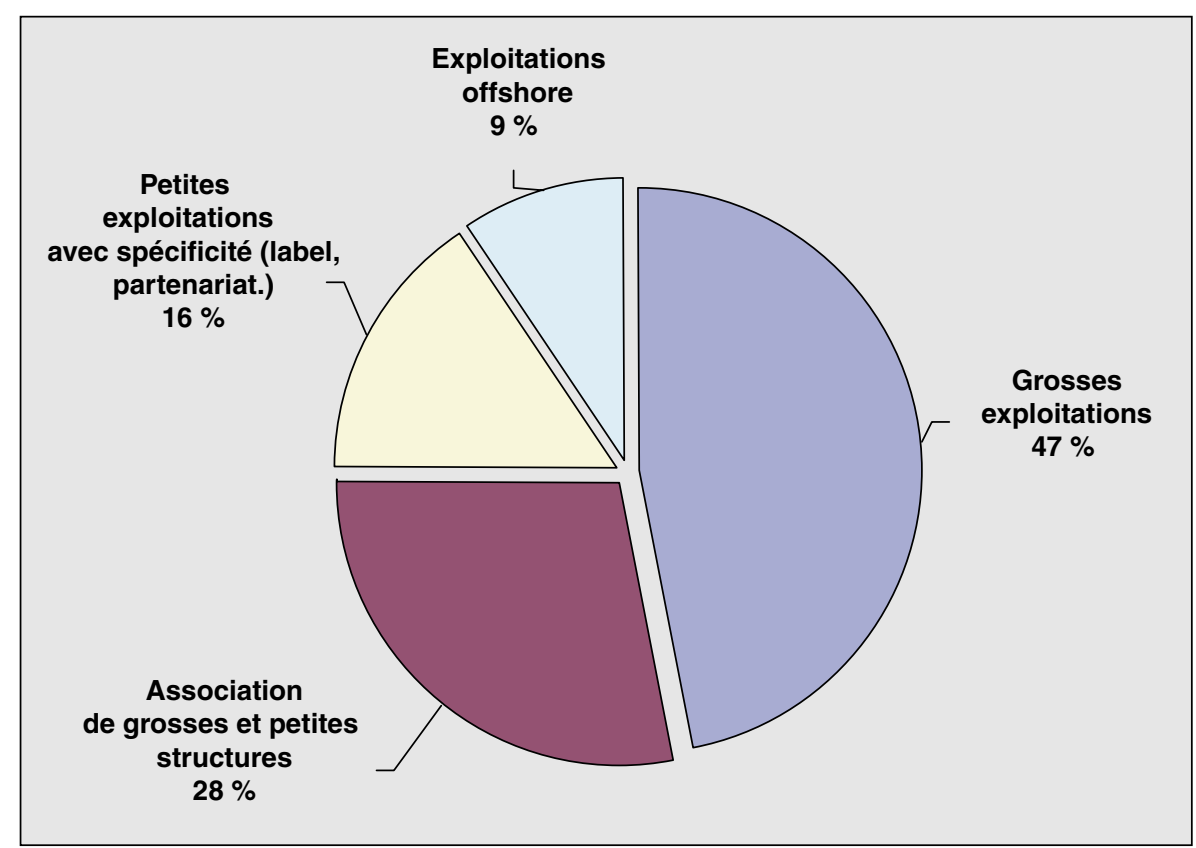

Figure 9. Perspectives d'évolution de la structure économique des exploitations.

Figure 9. Possible evolution of economic structures Source : enquêtes EVAD, France et Chypre été 2006. diversité fonctionnelle des exploitations qui soit fonction des contextes locaux dans lesquels l'activité doit s'intégrer au mieux. Cette évolution vers une aquaculture durable suppose non seulement des évolutions des pratiques d'élevage, mais aussi comme on l'a souligné et comme l'évoque une grande partie des acteurs enquêtés, de renforcer la structuration du secteur et sa politique de communication. Ce renforcement institutionnel du secteur va dans le sens des recommandations du projet de protocole pour la mise en œuvre de la gestion intégrée en Méditerranée, qui insiste sur la coordination institutionnelle, la participation et la formation des acteurs. La méthodologie que nous avons utilisée pour analyser la représentation que les acteurs ont ou se font du développement durable peut être utilisée dans d'autres situations (pays) de la Méditerranée pour compléter ce bilan. Un regard particulier devra être porté sur la distance qui sépare le fonctionnement actuel des unités de production de celui qu'“ impose " la prise en compte du développement durable qui est une des conditions essentielles de son développement à venir.

\section{Références}

Barnabé G, Rene F. Reproduction contrôlée et production d'alevins chez la Dorade Sparus auratus Linne 1758. CR Hebd Seances Acad Sci Paris (D) 1973 ; (276) : 1621-4.

Benoît G, Comeau A. Méditerranée, les perspectives du Plan Bleu sur l'environnement et le développement. Paris: éditions de I'Aube, 2005.

Clément $O$. Un outil pour la construction d'indicateurs de développement durable: la méthode Delphi. Une expérience en Aquaculture. Natures Sciences Sociétés $2006 ; 14: 6$.

Délégation interministérielle à l'aménagement et à la compétitivité des territoires (Diact). Rapport français d'application de la Recommandation du parlement européen et du Conseil du 30 mai 2002 relative à la mise en œuvre d'une stratégie de gestion intégrée des zones côtières en Europe. Paris : Diact éditins, 2006.

EI Sadeck S. Guide on the aquaculture interaction with environment in the mediterranean sea (Egypt case). Communication à la reunion FEAP/IUCN Framework for sustainable development of aquaculture. Guide on the interacpment of aquaculture. Guide on the interac-
tion with the environment, Grand Canaria, 26-28 October 2006. Malaga : UICN Méditerranée, 2006.

Gafsi M. Exploitations agricoles et agriculture durable. Cah Agric 2006 ; 15 : 491-7.

Lazard J, Aubin J, Clément O. Le développement durable de I'aquaculture. CR Acad Agric Fr 2005; 91 (séance du 12 octobre 2005): 33-43.

Figure 10. Possible evolution of farming techniques. Source : enquêtes EVAD, France et Chypre été 2006. 
Mathé S, Brunel O, Rey-Valette H, Clément O. Recensement des initiatives en faveur de la durabilité de l'aquaculture. Rapport CEP/UICN-EVAD. Malaga: UICN Méditerranée, 2006.

Programme d'action méditerranéen (PAM) : Progamme des Nations unies pour l'environnement (Pnue). Rapport de la deuxième réunion du groupe de travail d'experts désignés par les parties contractantes sur le projet de protocole relatif à la gestion intégrée des zones côtières. Loutraki (Grèce), 6-9 septembre 2006. WG 298/4. Athènes: Plan d'action pour la Méditerranée (PAM) : Programme des Nations unies pour l'environnement (Pnue), 2006.
Rey-Valette H, Roussel S. L'évaluation des dimensions territoriale et institutionnelle du développement durable : le cas des politiques de Gestion Intégrée des Zones Côtières (GIZC). Revue Développement Durable et Territoire $2006 ;(8)$ : p. 20 (revue électronique).

United Nations Food and Agriculture Organisation (FAO). Aquaculture production : quantities 1950-2004. Rome: FAO, Fishery Information, Data and Statistics Unit, 2004.

University of Stirling. Study of the market for aquaculture produced seabass and seabream species. Report to the European commission $D G$ fisheries. Stirling (Grande-Bretagne) : University of Stirling, 2004.
Union internationale de conservation de la nature (UICN). Minutes of the workshops on aquaculture held within the framework of the World Conservation Forum. Bangkok, 17-25 November 2005. Malaga : Rome : UICN Méditerranée ; organisation des Nations unies pour I'alimentation et I'agriculture (FAO) ; Fédération européenne des producteurs aquacoles (FEAP), 2005a.

Union internationale de conservation de la nature (UICN). Développement durable de l'aquaculture méditerranéenne. Conclusions de l'atelier de Sidi Fredj, Alger, 25-27 juin, 2005. Malaga : UICN Méditerranée, 2005b. 